\title{
MEASUREMENT OF THE ERROR IN WILSON'S CENTRAL TERMINAL: AN ACCURATE DEFINITION OF UNIPOLAR LEADS
}

\author{
BY \\ G. E. DOWER, J. A. OSBORNE, AND A. D. MOORE \\ From the Departments of Pharmacology, Medicine, and Electrical Engineering of the University of \\ British Columbia, Canada* \\ Received June 16, 1958
}

Unipolar leads using Wilson's central terminal as an "indifferent" electrode have been in general clinical use for many years. The central terminal was not introduced as a theoretically correct reference for unipolar leads, but as a convenient reference that was at least better than using one of the limbs alone, as in the old CR, CL, and CF leads, in which the exploring electrode potentials were compared with those of electrodes placed respectively on the right arm, the left arm, or the left leg (Wilson et al., 1934). When unipolar leads are used to explore the potentials over the left chest at the level of the heart, the error in adopting the central terminal as a reference electrode is small because the potential variations of the exploring electrode are relatively large. A tendency to explore the right chest has developed in recent years. In this case, the error may be considerable because the smaller potential variations of the exploring electrode may be of a magnitude comparable with those of the central terminal itself. Such records may be open to misinterpretation. A case has recently been described in which the unipolar lead tracings V3R were produced by potential variations of the central terminal rather than by those of the exploring electrode (Osborne and Dower, 1957), and resulted in a pattern resembling right ventricular hypertrophy for this lead.

Methods of determining the error in the central terminal have been described by Eckey and Fröhlich (1938), Burger (1939), Wilson et al. (1949), Dolgin et al. (1949), Bayley and Kinard (1954), and Frank (1955a), but none has been applicable to patients. For this reason, it has not been possible to study the error under clinical conditions. The method to be described makes this possible. The theoretical considerations that arose out of the development of this method have demanded a careful scrutiny of previous definitions of unipolar leads and this has led unexpectedly to the conclusion that a more accurate definition is required.

\section{Principle}

The electrical potential at a point, A, with respect to some other point, B, may be defined as the increase in potential energy that a unit positive charge will experience when moved from B to A. Just as, in mechanics, the potential energy of a body (in a uniform gravitational field) is determined by the height through which it is raised, and heights can only be measured with respect to some level of reference, so electrical potential requires a reference for its measurement: only potential differences have meaning. Since the field of a system of charges confined to a finite region of space vanishes at infinity (Stratton, 1941), it is customary to consider the absolute potential of a point as its potential with respect to infinity.

Let us imagine the subject to be in free space, and let the net charge on his body be zero. What

* This work was aided by grants from the National Research Council of Canada and the British Columbia Heart Foundation. 
factors affect the potential of a point on the surface of the body with respect to infinity? One factor is certainly the heart itself and another is the configuration of the various conducting media, or tissues, of the body; together, these factors determine the potential differences between points on the body surface. The absolute potentials of these points are subject to another influencethe external field distribution which is related to the capacitance between various regions of the body and infinity. (The capacitance, $C$, of a body is the proportionality constant relating the potential, $v$, and the electric charge, $q$, i.e. $v=\frac{q}{C}$.) This is illustrated in Figure 1. The subject is
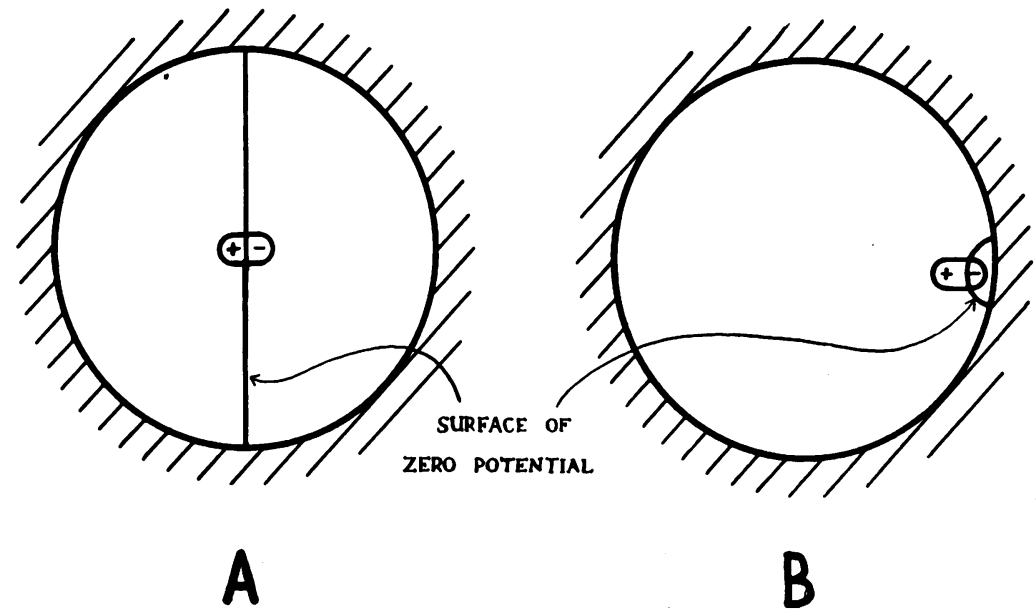

FIG. 1.-(A) Fusiform body at the centre of a large, hollow, spherical conductor. The net charge on the body and the net charge on the conductor are zero. A "cardiac" generator at the centre of the body produces a charge separation, making one end instantaneously positive and the other negative with respect to the surface of zero potential. Note that the surface of zero potential, which represents the potential of the sphere, lies in the equatorial plane. (B) When the body is moved to an eccentric position, the surface of zero potential no longer lies in the equatorial plane of the body. The potential of points on the surface of the body with respect to the sphere has thus been altered.

represented as a fusiform body symmetrical about its equatorial plane. Infinity may be considered as a sphere of infinite radius concentric with the body: we shall consider the body to be at the centre of a large conducting sphere (Fig. 1A). Let the net charge on the body be zero. Let us now say that, due to the action of a "cardiac" generator acting at the centre of the equatorial plane of the body, a time-varying separation of charge is brought about so that when one end of the body acquires a positive charge the other end of the body acquires an equal negative charge. Since the charge on one half is equal to that on the other, and the capacitances of the two halves with respect to "infinity" are equal (from symmetry), the potentials of the two ends are always numerically equal, being equally displaced from the reference, but in opposite senses.

Let us now move the body from its centric position so that one end approaches the wall of the conducting sphere (Fig. 1B). This will result in a disproportionate increase in the capacitance of that end with respect to the sphere. This increase in capacitance of one end is associated with a decrease in the amplitude of the potential variations of that end with respect to the sphere (writing $v=\frac{q}{C}$ for this end, we note that increasing $C$ reduces $v$ ). Thus, as one end of the body approaches the sphere, its potential variations with respect to the sphere diminish. But the original symmetry of capacitances could have been disturbed by means other than moving the body away from the centre of the sphere. For example, destroying the physical symmetry about the equatorial plane by attach- 
ing an appendage to one end of the body would lead to an inequality of capacitances. Even if such an appendage did not affect the electric field within the body, it would still alter the potentials of points on the body with respect to the sphere. It follows that, for a subject in free space, the potential of a region of his body is affected by the capacitance of that region, and the capacitances of all other regions of his body, with respect to infinity.

Because an increase in the capacitance with respect to infinity of some region of the body, such as we have considered, is associated with a corresponding disturbance of the electric field outside the body (compared with that existing for the body in free space), we might say that the potential of a point on the body is determined by the electric fields inside and outside the body. It happens that the electric field inside the body is relatively insusceptible to change from external factors (though it has been appreciably changed in some immersion experiments, e.g. Dolgin et al., 1949), but the electric field outside the body is highly susceptible to change from such factors. The definition of a unipolar lead used in this paper requires electric field distributions similar to those existing if the subject were in free space.

The conditions of free space may be approximated by placing the subject at the centre of a large hollow conductor, such as a shielded room, but insulated from it. If the room is large enough, the electric field near the body will not be sufficiently distorted from its configuration in free space for the results to be seriously affected. In addition to the room's being sufficiently large, its shielding must be adequate to prevent interference from currents induced from outside sources.

It can be shown that the potential of the shielded room with respect to infinity is unaffected by the action of the subject's heart within the room.* It is possible that agencies external to the shielded room may cause its potential to vary with respect to infinity, but we are concerned only with those within the room, which will have no effect. The walls of the room may thus be considered a legitimate substitute for a concentric sphere of infinite radius (infinity).

Let us assume that there be applied to the subject a resistance network having a reference terminal whose potential variations with respect to the walls of the shielded room, due to the action of the heart, can be reduced approximately to zero by suitable adjustments of variable components in the network. $\dagger$ (It is assumed that the capacitance of the network to the walls of the room is negligible.) The problem now becomes: How can we tell when the network is correctly set?

If a sensitive current-detector could be connected between the reference terminal of the network and the shielding of the room without affecting the electric field, the correct adjustment would be indicated by the condition of zero current flow between the subject and the shielding (the circuit being completed by the capacitance between them). At this setting, because there would be no flow of current through the connecting wire, it could be considered as an open circuit, i.e.connecting the patient to the shielded room would not affect the potentials at his body surface with respect to the shielding. Further, if the connecting wire were then removed, the potential of the reference terminal would remain at that of the shielding. If the potential of the shielding could be taken for our purposes to be that of an electrode at infinity, we might say that the network had been correctly adjusted to give a reference terminal whose absolute potential was zero. It might be asked: Why should it be necessary to obtain a reference terminal to substitute for the shielded room? Why could not the shielding alone be used as a reference? Actually, it can and, indeed, the adjustable network was not used for most of the determinations of the error in Wilson's central terminal. However, the use of such a network brings certain advantages which will be mentioned later.

\section{METHOD}

The shielded room measured $3 \times 2.7 \times 3 \mathrm{~m}$. Shielding was obtained by applying a $0.25-\mathrm{mm}$. layer of tin-plate to its inner surfaces, except on the floor where a 1-mm layer of galvanized iron was used. The sheets

* This is an application of Gauss's flux theorem which states that the surface integral of the flux density, D, over any closed surface, $\mathbf{S}$, is equal to the algebraic sum, $q$, of all the charges inside the surface, or $\oint \mathbf{D} \cdot \mathbf{d S}=q$.

$\dagger$ Justification for the assumption that, for any fixed position of the "electrical centre" of the heart, there exists a network with a central terminal that does not vary due to the electrical activity of the heart may be found in the work of Frank (1955b). 
of tin-plate overlapped at their edges, which were soldered together at intervals of about $5 \mathrm{~cm} . \quad$ A similar covering was applied to the doors; when the doors were shut there was a spring contact between the tin-plate on the doors and on the walls of the room. Lighting was provided by a battery lantern-there were no windows or electrical outlets.

A hammock was strung between opposite corners of the room and the foot-end could be raised or lowered with a block and tackle. The loose hammock was draped over a chair and the patient seated upon it. The block and tackle were then used to raise him to the centre of the room.

An adjustable reference terminal was attached to the patient by four electrodes situated as follows: the right arm, R; the mid-axillary line at the level of the 5th chondrosternal junctions, A; the left leg, F; and the anterior mid-line, E, at the same level as A.* The R and A, and F and E electrodes were respectively connected to the ends of a pair of potentiometers, I and II, the sliders of which were themselves connected to the ends of a third potentiometer, III. The slider of III formed the reference terminal of the network. The three potentiometers were each of $100 \mathrm{kilohms}$ and were mounted immediately over the patient. They were adjustable from outside the room by turning three glass rods which pierced the wall.

The adjustable reference terminal, shown diagrammatically in Fig. 2, was directly attached to the grid of an electrometer tube (Victoreen 5803) which, because of its low grid current, permitted the use of a large grid-leak resistance (1000 megohms). The electrometer tube was mounted on the wall of the room. It was operated as a class-A amplifier and provided a voltage amplification of 1.5. The time-constant of the system was about $\mathbf{0 . 0 5}$ second, which was sufficient for amplification of the QRS complex. The error in Wilson's central terminal when the $T$ waves were being inscribed was not studied.

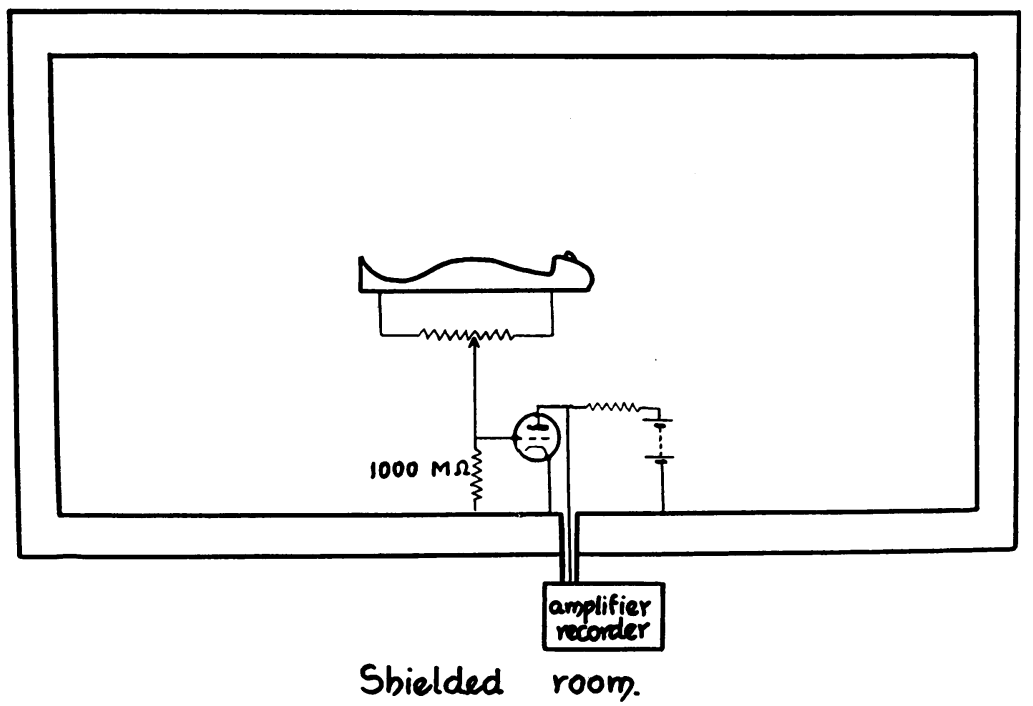

Fig. 2.-Patient at the centre of shielded room. The adjustable reference terminal is represented as the slider of a single potentiometer (though three were actually used). The battery-operated electrometer amplifier is shown within the room connected to a line-operated amplifier and recorded outside.

Further stages of amplification were obtained from a line-operated amplifier (Sanborn Twin Beam) placed outside the room. Visible records were immediately available from an oscillograph (Heiland Visicorder). The over-all sensitivity was $5.6 \mathrm{~cm}$. $/ \mathrm{mv}$.; the best paper speed was $125 \mathrm{~mm}$./sec.

The basic noise level was determined by attaching the electrometer input to a large sheet of tin-plate placed in the hammock: it was found to be 10 microvolts. The resistances of the hammock and supports were roughly determined with a megger. The resistance of the supports was found to be greater than $10^{12} \mathrm{ohms}$ and the resistance of the hammock was found to be of the same magnitude.

Ballistic artifact. The system was found to be extremely sensitive to movement of static charge within

* These electrode positions are those used in a recently described VCG lead system, the RAFE (Dower and Osborne, 1958). The exact positions are not important, however, so long as they embrace the origin of image space. 
the room. For example, with the electrometer input attached to the piece of tin-plate, as described above, the gentle swinging of a rubber strap (of the kind used to secure the electrodes on the limbs) was easily detected, even though it was several feet away from the tin plate. This effect was very troublesome when a patient was attached to the input. It was necessary to support him on an insulator, yet any insulator possesses some static charges, and relative movement between patient and support generated signals several orders of magnitude larger than those being studied. After many trials, a canvas hammock was found the best solution to this problem. It was also necessary to remove clothing of nylon or similar material and dissipate static electricity on the patient and hammock through a grounded soft wire brush. In spite of these measures, it was not possible to remove the ballistic artifact altogether, but in nine out of ten patients it no longer interfered with the determination of a null setting of the potentiometers, and signals greater than 1/50 of a millivolt could be detected, particularly at the paper speed mentioned above.

Adjusting the network to a null. With the patient quietly at rest in the hammock, the static discharging procedure completed, the $\mathrm{R}, \mathrm{A}, \mathrm{F}$, and $\mathrm{E}$ electrodes attached to the patient and connected to the potentiometers, and all extraneous material removed from the room, the door was closed and the patient was left alone. Potentiometers I, II, and III were adjusted in turn until the amplitude of the QRS pattern was a minimum. By this means it was sometimes possible to remove all trace of the QRS (signal less than $0.02 \mathrm{mv}$ ); at other times it was not possible to obtain a reference terminal that varied, during the QRS interval, by less than $0 \cdot 1 \mathrm{mv}$. The reference obtained was, of course, unique for each patient.

Effect of room size. In order to determine whether the room used was large enough, its effective size was reduced by large metal screens measuring $1.2 \times 2.4 \mathrm{~m}$. which were placed on each side of the patient at equal distances away. These were gradually brought closer to the patient and the effect on the null setting of the potentiometers was noted. No appreciable effect was observed on bringing the screens to a distance of one metre from the patient. When the screens were brought much closer than this the ballistic artifact became too great for clear interpretation of the results. It was concluded that the room was larger than required in so far as producing negligible distortion of the electric field near the body was concerned but that a room of much smaller dimensions would have greatly increased the ballistic effects.

Effect of connecting detector to reference terminal of network. Theoretically it can be shown that attaching a wire to the reference terminal of the network and leading this to the current-detecting device on the wall of the shielded room will distort the electric field near the body and, therefore, change the potential of the reference terminal. It can be argued that if such a distortion were appreciable, bringing the lead wire in towards the patient from another direction might produce a somewhat different distortion and hence a somewhat different record. To study this, the lead wire was brought to the patient from various directions that were as different as possible. Sometimes it approached the patient perpendicularly and sometimes it ran parallel to the surface of the patient, only a few centimetres away, for distances up to one metre. None of these measures had a noticeable effect on the records obtained. It was concluded that the effect of connecting the detector to the reference terminal of the network was insignificant.

Wilson-central terminal comparison. When the network had been adjusted to give a null, its reference terminal was used for measuring the potential of Wilson's central terminal. The patient was grounded via the reference terminal of the network, and Wilson's central terminal was attached to the input of the amplifier. As impedances were then low (the patient was grounded) no ballistic artifact was encountered and tracings such as that illustrated in Fig. 3A were obtained. An example of ballistic artifact is shown in Fig. 3B.

Direct measurement of the potential of Wilson's central terminal. It has already been pointed out that the adjustable network is not needed and that the potential of Wilson's central terminal could be directly measured with respect to the walls of the room, the electrometer amplifier being used as a voltmeter. This necessitated calibration of the amplifier-recording system. The calibration was carried out by a comparison of the amplitude of the signals obtained when the potential variations of Wilson's central terminal were measured directly and indirectly, i.e. using the reference terminal of the adjustable network. The amplitude turned out to be the same in both cases.

Measurements of the potential variations of Wilson's central terminal with respect to the walls of the shielded room, during the QRS interval, in 117 patients are shown in Table I. The mean potential was $0.09 \mathrm{mv}$ and the variation was -0.3 to $+0.4 \mathrm{mv}$. If a Gaussian distribution is assumed, there is a 26 per cent probability of the central terminal deviating from zero by $0.2 \mathrm{mv}$ or more. (The table indicates that 52 subjects showed this variation, but the figures are rounded off; in fact, 23 of these had variations between 0.15 and 0.2 . This leaves 29 out of 117 , or 25 per cent, who showed variations equal to, or greater than, $0.2 \mathrm{mv}$ ). There is an 8 per cent probability of the deviation being greater than $0.3 \mathrm{mv}$. 


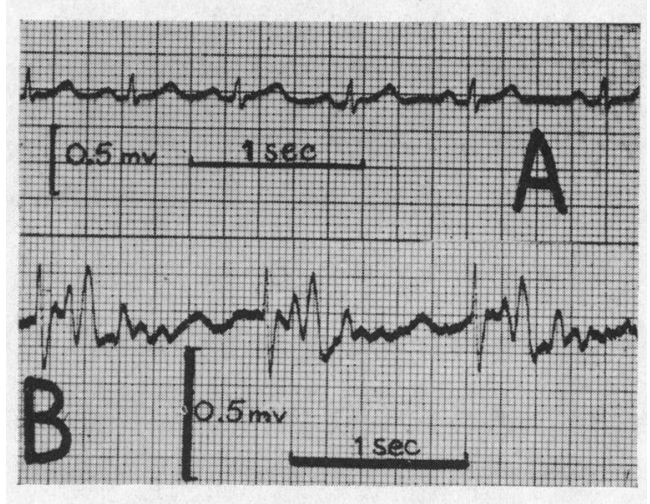

FIG. 3.-(A) The error in Wilson's central terminal recorded as the potential difference between the central terminal and an accurately adjusted reference terminal (see text). (B) An example of ballistic artifact encountered when a single wire led from the ungrounded patient to the electrometer amplifier.

TABLE I

The QRS-Potential Variations of Wilson's Central Terminal with Respect to the Walls of a SHIELDEd RoOM, in 117 SUbJects

\begin{tabular}{c|c}
\hline $\begin{array}{c}\text { Potential variation } \\
\text { in millivolts }\end{array}$ & $\begin{array}{c}\text { Number of subjects } \\
\text { with each potential } \\
\text { variation }\end{array}$ \\
\hline$-0 \cdot 3$ & 2 \\
-0.2 & 6 \\
-0.1 & 13 \\
0 & $19 *$ \\
$+0 \cdot 1$ & 33 \\
$+0 \cdot 2$ & 30 \\
+0.3 & 12 \\
+0.4 & 2 \\
Arithmetical mean +0.09 & \\
\hline Standard deviation 0.15 & \\
\hline
\end{tabular}

* Includes all those in whom ballistic artifact precluded accurate determination.

\section{Discussion}

The definition of a unipolar lead used in this paper is as follows: a unipolar lead is one in which the potential variations that develop between a single exploring electrode and an electrode at an infinite distance are recorded, with the subject in free space. This definition ensures that the electric field within and without the surface of the body are normal, i.e. unaffected by extraneous influences. Unipolar potentials are thus absolute in the sense in which the term is used in physics.

One of the original definitions put forward by Wilson et al. (1931) for a true unipolar lead was that it gave "the potential at any point on the body at any instant with reference to the potential of this point at a time when the heart is producing no electric currents, a potential which we may for our purposes regard as zero." Actually this does not define a unique reference for, if any point on the body is connected to the ground and the earth is used as a reference, the potential of such a point will remain zero whether the heart is beating or not. If the heart is considered to be the only agent producing charge separation in the body, when it is inactive all points on the body will be at at the same potential: that of the earth. Suppose the left leg is connected to earth: when the heart 
is producing no electric currents, the potential of any point on the body will be that of the left leg and, therefore, of the earth. If the potential of an exploring chest electrode is now measured with respect to ground, it may also be considered to be measured with respect to the potential such an electrode would have when the heart was producing no electric currents. According to the above definition, the potentials measured should be unipolar lead potentials. But, in fact, we do not have a lead that is any different from one in which the left leg is used as a reference: the lead is not unipolar, but bipolar. This difficulty is resolved, however, if the electric field external to the body is specified. If the field is considered to be that existing in free space (and there seems to be no reason for considering it to be different from this) Wilson's definition becomes equivalent to ours.

It might be held that true zero should be defined as the potential that Wilson's central terminal would have if the assumptions in Einthoven's triangle scheme were, in fact, correct (Burger, 1939). According to Einthoven (cited by Wilson et al., 1946), "the triangle was supposed to be a homogeneous sheet of conducting material and in regard to the distance between the two points of the bipole, of a large, let us say infinite, extent." From symmetry, the potential of the central terminal will be the same as that of the plane of symmetry passing through the bipole (separating positive and negative isopotentials). But this plane reaches to infinity, so the central terminal and the electrode at infinity lie on the same isopotential surface, i.e. they are at the same potential. Wilson et al. (1946), speaking of experiments in which the subject was placed in a liquid of lower conductivity than the body, said: "If the conductivity of the isolated conductor (the body) under consideration is large enough, we may think of it as in contact over its whole surface with an infinite conducting medium possessing a very much smaller conductivity and thus make it possible, at least theoretically, to choose infinity as our reference point for the measurement of the field." The immersion experiments require that the subject be in a medium of much lower conductivity than the body tissues, such as distilled water (Eckey and Fröhlich, 1938); otherwise short-circuiting will appreciably reduce body potentials (Wilson et al., 1946; Dolgin et al., 1949; and Bayley and Kinard 1954). If the conductivity of the medium is reduced to the limit, the immersion experiments become identical with the field experiments. It should be noted that in the immersion experiments it is still necessary to specify the electric field distribution external to the body because conductivity of the medium close to the body must be uniform, just as the dielectric must be uniform in the field experiments. Further, attaching a conducting plate to some part of the body would affect the result in both types of experiments. To avoid disturbance of the electric field near the body, therefore, the subject should be in a large tank, just as he should be in a large shielded room in the field experiments. In the experiments of Eckey and Fröhlich the tank used was very small. In the experiments of Bayley et al. (1954) the subject was placed in a spherical integrating electrode six feet in diameter: the fact that this sphere was a conductor must have appreciably changed the electric field produced by the heart; it also rendered inapplicable some of the equations adduced in support of the method.

A definition of zero is implied when unipolar electrocardiograms are derived graphically from corresponding vectorcardiograms (Osborne and Dower, 1957). The zero reference in this case is the origin, and the cathode-ray beam is at the origin when no signals are being applied to the deflection plates of the cathode-ray tube, i.e. when the heart is producing no electric currents. At this time the electric fields inside and outside the body are zero. Because it is zero, the external electric field will not be distorted by any wires connecting the patient to ground. In fact, because there would be no current flowing in such wires, they may be considered open circuits, i.e. the patient is effectively in free space. Thus the implied definition of unipolar potentials is identical with that used in this paper.

Unipolar lead potentials have been defined by Burger and van Milaan (1948) in terms of image space. They may be expressed as $v=\mathbf{L} \cdot \mathbf{H}$, where $v$ is the potential of the exploring electrode, and $\mathbf{L}$ is the corresponding lead vector that must be used to obtain $v$ from $\mathbf{H}$, the instantaneous heart vector. Since the reference of potential is not stated, it will be taken as the potential of an electrode at infinity. With the subject in free space, we may say that for every point in real space there is an 
instantaneous potential, $v$, due to $\mathbf{H}$. The above relationship between $v$ and $\mathbf{H}$ thus defines vectors, $\mathbf{L}$, the tips of which define points in image space corresponding to points in real space where $v$ is measured. The potentials recorded at all points on the surface of the body thus define a set of points in image space: this set forms the image surface. Now, as an exploring electrode approaches the heart in real space, $v$ generally becomes larger, i.e. the magnitude of $\mathbf{L}$ increases, so that points inside the body surface correspond to points outside the image surface, and vice versa. But, from our definition of zero, $v=0$ for all directions of $\mathbf{H}$ for an electrode at infinity. This can only happen when $\mathbf{L}=0$, i.e. at the origin of image space. Hence, infinity in real space corresponds to the origin of image space. This justifies the choice of the potential of an electrode at infinity as a reference. It should be noted that it is not necessarily consistent to consider that the midpoint of the cardiac dipole corresponds to the origin of the image space, as some accounts suggest (Frank, 1954).

It has already been explained that, with the subject in free space, the absolute potential variations of points on the body due to a time-varying generator will be influenced by the capacitances of the various regions of the body with respect to infinity. Hence, unipolar potentials as defined in this paper are subject to three variables: the heart itself, the conductivities of the body tissues (which largely determine the configuration of the electric field within the body), and the regional capacitances (which largely determine the configuration of the electric field outside the body). A consequence of this is that a part of the body, such as a leg, might be removed, and cause little disturbance of the electric field within the body, but because of its effect on the external electrical field, its removal would change the unipolar electrocardiogram. The objection might therefore be raised that the proposed definition has introduced an unnecessary variable. In fact, such a complication is not unnecessary-it is a consequence of the concept of unipolar leads. Some authors prefer to think of the problem of finding a reference for unipolar leads as being confined to the body itself, since with the subject in air the electric field distribution within is not appreciably disturbed by conditions without. If this is done, it becomes necessary to define a reference of potential inside the body. The theoretical reference for unipolar leads that has been suggested is the "dipole mid-potential" (Burger, 1939; and Frank et al., 1955). Unfortunately, a point representing the dipole mid-potential cannot be defined in the body, though it can in idealizations such as Einthoven's triangle. If the heart is represented electrically as an infinitesimal dipole in an infinite homogeneous medium, the dipole mid-potential may be considered as the potential at the plane of symmetry of the dipole. Since this plane reaches to infinity, the potential of this reference is identical with that of an electrode at infinity. But a difficulty arises when the heart is represented by a dipole, either finite or infinitesimal, in the body. In this case, the use of the dipole mid-potential is inexact because, if the dipole is finite, the electrical and geometrical midpoints are not necessarily identical, while, if it is infinitesimal, the plane of symmetry is not an isopotential. It should also be pointed out that the use of the dipole concept in the definition of unipolar leads requires that the heart can be represented by a dipole (which is not a matter of uniform agreement), whereas the definition adopted in this paper makes no such assumption.

When the results obtained with the present technique are compared with those of previous workers it should be noted that in the experiments in which the error in Wilson's central terminal was determined by immersing the subject in water, in addition to the practical inconvenience of this procedure, difficulty was generally encountered from short-circuiting, which greatly reduced the measurable error. When distilled water was used, and short-circuiting was minimal, errors up to $0.3 \mathrm{mv}$ were recorded-similar in magnitude to those obtained with the present technique, in which short-circuiting was no problem.

The implication arising out of the present work is that unipolar lead QRS signals of less than $0.3 \mathrm{mv}$ should be interpreted with caution.

\section{SummaRY AND CoNClusions}

The realization that error in Wilson's central terminal could lead to clinical misinterpretation of electrocardiograms has prompted the development of a method for measuring this error in patients. 
The patient is placed in a hammock at the centre of a shielded room and the potential between the central terminal and the shielding measured directly. Development of the principle underlying this technique has necessitated the adoption of a more precise definition of zero reference than that originally proposed by Wilson and his associates. The potential of an electrode at an infinite distance is taken as zero and the configuration of the electric field inside and outside the body is considered to be that which would exist if the body were in free space. This more complete definition has been shown to be consistent with the general concept of unipolar leads.

The error in the central terminal was measured in 117 cases and found to be greater than $\pm 0.2 \mathrm{mv}$ in 29 . There appeared to be an 8 per cent probability that the error would, in any given subject, exceed $0.3 \mathrm{mv}$. It is concluded that electrocardiographic diagnosis should not hinge on standard unipolar lead QRS signals of this magnitude.

The advice of Dr. J. G. Foulks and the assistance of Dr. O. Papp are gratefully acknowledged.

\section{REFERENCES}

Bayley, and Kinard, C. L. (1954). Circulation Res., 2, 104.

- , R. M., Reynolds, E. W., Kinard, C. L., and Head, J. F. (1954). Circulation Res., $2,4$.

Burger, H. C., and van Milaan, J. B. (1948). Brit. Heart J., 10, 229.

Burger, R. (1939). Cardiologia, 3, 56.

Dolgin, M., Grau, S., and Katz, L. N. (1949). Amer. Heart J., 37, 868.

Dower, G. E., and Osborne, J. A. (1958). Amer. Heart J., 55, 523.

Duchosal, P. W., and Sulzer, R. (1949). La Vectocardiographie. Basel, S. Karger.

Eckey, P., and Fröhlich, R. (1938). Arch. Kreislaufforsch., 2, 349.

Frank, E. (1954). Amer. Heart J., 47, 757.

- (1955) Amer. Heart J., 49, 670.

- (1955). Circulation, 11, 937.

- Kay, C. F., Seiden, G. E., and Keisman, E. A. (1955). Circulation, 12, 406.

Osborne, J. A., and Dower, G. E. (1957). Amer. Heart J., 54, 722.

Stratton, J. A. (1941). Electromagnetic Theory. New York, McGraw-Hill Book Co. Inc.

Wilson, Bryant, J. M., and Johnston, F. D. (1949). Amer. Heart J., 37, 493.

- Johnston, F. D., Macleod, A. G., and Barker, P. S. (1934). Amer. Heart J., 9, 447.

,-- Rosenbaum, F. F., and Barker, P. S. (1946). Amer. Heart J., 32, 277.

Z, Macleod, A. G., and Barker, P. S. (1931). Amer. Heart J., 7, 207. 